\title{
Diophantine Approximation, Lagrange and Markov Spectra, and Dynamical Cantor Sets
}

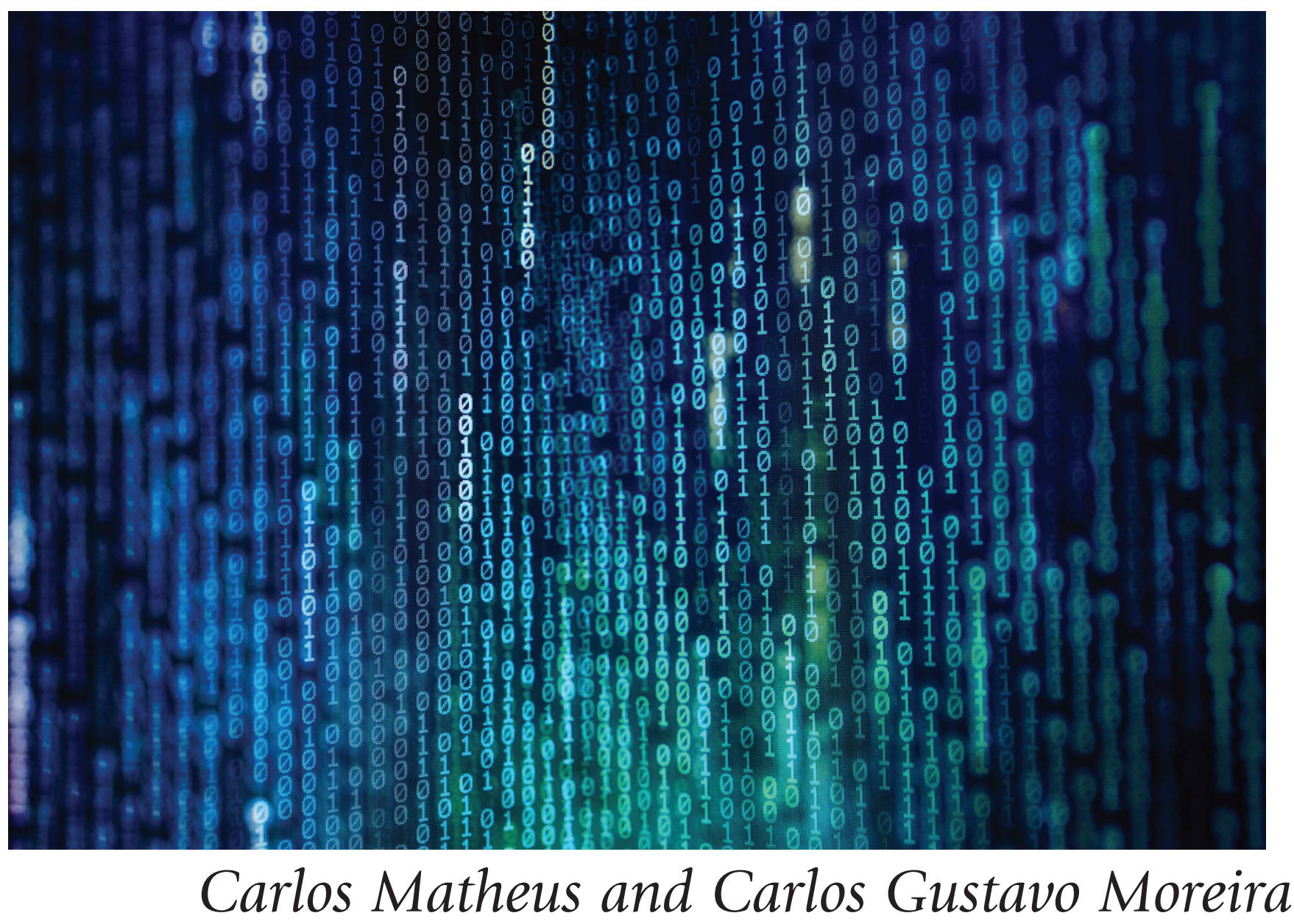

\section{Diophantine Approximation}

The seminal works of Diophantus of Alexandria (circa AD 250) on rational approximations to the solutions of certain algebraic equations began the important subfield of

Carlos Matheus is a CNRS researcher affiliated to École Polytechnique (France). His email address is carlos . matheus@math.cnrs.fr.

Carlos Gustavo Moreira is a full professor at IMPA (Brazil). His email address is gugu@impa.br.

Communicated by Notices Associate Editor William McCallum.

For permission to reprint this article, please contact:

reprint-permission@ams.org.

DOI: https://doi.org/10.1090/noti2329 number theory called Diophantine approximation. Among the basic problems in this topic, one has the question of finding rational numbers $p / q \in \mathbb{Q}$ approximating a given real number $\alpha \in \mathbb{R}$ in such a way that the denominator $q$ is not "big" and the error $|\alpha-p / q|$ is "small."

1.1. Rational approximations of $\pi$. The first few decimal digits of the number $\pi$ are well known: $\pi=3.1415926 \ldots$. By definition, this provides some rational approximations of $\pi$ like $314 / 100$ and $3141592 / 10^{6}$. Nonetheless, these fractions are certainly not answers to the Diophantine problem posed above because we can get better approximations with smaller denominators: for instance, 
Archimedes (circa 250 BC) knew that

$$
\left|\pi-\frac{22}{7}\right|<\frac{1}{700}<\left|\pi-\frac{314}{100}\right|
$$

and it is possible to check that

$$
\left|\pi-\frac{355}{113}\right|<\frac{1}{3 \cdot 10^{6}}<\left|\pi-\frac{3141592}{10^{6}}\right| .
$$

1.2. Dirichlet's pigeonhole principle. The example of the number $\pi$ makes us wonder how small $|\alpha-p / q|$ can be when the denominator $q$ varies in a fixed range $1 \leq q \leq Q$. A preliminary answer comes from the following elementary remark. Recall that any real number $\alpha$ lies between two consecutive integers, namely $\lfloor\alpha\rfloor \leq \alpha<\lfloor\alpha\rfloor+1$, where $\lfloor\alpha\rfloor \in \mathbb{Z}$ is the integer part of $\alpha$. Therefore, given $\alpha \in \mathbb{R}$ and $q \in \mathbb{N}$, we can find $p \in \mathbb{Z}$ such that $|q \alpha-p| \leq 1 / 2$, i.e.,

$$
\left|\alpha-\frac{p}{q}\right|<\frac{1}{2 q} \text {. }
$$

In 1841, Dirichlet used his famous pigeonhole principle to significantly improve upon the elementary statement in the previous paragraph: more concretely, for any irrational number $\alpha$, one has that

$$
\#\left\{\frac{p}{q} \in \mathbb{Q}:\left|\alpha-\frac{p}{q}\right|<\frac{1}{q^{2}}\right\}=\infty .
$$

Indeed, given $Q \in \mathbb{N}$, Dirichlet considered how the $Q+$ 1 numbers $\{k \alpha\}:=k \alpha-\lfloor k \alpha\rfloor \in[0,1), k=0, \ldots, Q$, are distributed across the elements of the partition $[0,1)$ into $Q$ equal intervals. By the pigeonhole principle, two fractional parts, say $\{k \alpha\},\{j \alpha\}, 0 \leq k<j \leq Q$, must lie in the same interval, say $[(n-1) / Q, n / Q)$, so that $|\{j \alpha\}-\{k \alpha\}|<1 / Q$ and, a fortiori, there exists $p \in \mathbb{Z}$ such that

$$
\left|\alpha-\frac{p}{j-k}\right|<\frac{1}{(j-k) Q} \leq \frac{1}{(j-k)^{2}} .
$$

1.3. Hurwitz theorem. In 1891, Hurwitz proved ${ }^{1}$ that Dirichlet's theorem is essentially optimal as far as all irrational numbers are concerned: one has

$$
\#\left\{\frac{p}{q} \in \mathbb{Q}:\left|\alpha-\frac{p}{q}\right|<\frac{1}{\sqrt{5} \cdot q^{2}}\right\}=\infty
$$

for all irrational numbers $\alpha$, and

$$
\#\left\{\frac{p}{q} \in \mathbb{Q}:\left|\frac{1+\sqrt{5}}{2}-\frac{p}{q}\right|<\frac{1}{(\sqrt{5}+\varepsilon) \cdot q^{2}}\right\}<\infty
$$

for all $\varepsilon>0$.

\section{Classical Spectra}

Despite the almost optimality of Dirichlet's theorem, we can ask whether it can be improved for individual irrational numbers $\alpha$ by inquiring about the nature of the best constant $\ell(\alpha)$ among the quantities $c$ such that

$$
\#\left\{\frac{p}{q} \in \mathbb{Q}:\left|\alpha-\frac{p}{q}\right|<\frac{1}{c \cdot q^{2}}\right\}=\infty,
$$

\footnotetext{
${ }^{1}$ Actually, this result was first established by Korkine and Zolotarev in 1873.
}

i.e.,

$$
\ell(\alpha):=\limsup _{p, q \rightarrow \infty} \frac{1}{q^{2}|\alpha-p / q|}=\limsup _{p, q \rightarrow \infty} \frac{1}{|q(q \alpha-p)|} .
$$

The Lagrange spectrum $L$ is the collection of finite ${ }^{2}$ best constants of Diophantine approximation, i.e.,

$$
L:=\{\ell(\alpha)<\infty: \alpha \in \mathbb{R} \backslash \mathbb{Q}\} .
$$

In this setting, the Hurwitz theorem says that the minimum of $L$ is $\sqrt{5}$. The Lagrange spectrum is an amazingly complex object. In this section we recount the history of results about it, including those indicated in Figure 1. We refer to the slightly expanded version of this survey article in arXiv:2105.01449 [math.NT] for a more detailed discussion on some of these results.

2.1. Beginning of the classical spectra. The Lagrange spectrum was systematically studied in connection with the theory of binary quadratic forms by Markov in 1879 . In fact, the quantity $q(q \alpha-p)$ is the value of the binary quadratic form $h_{\alpha}(x, y)=\alpha y^{2}-x y$ at the integral point $(p, q) \in \mathbb{Z}^{2}$, so that the Lagrange spectrum is somewhat related to the Markov spectrum $M$ of finite best constants

$$
m(h):=\sup _{(p, q) \in \mathbb{Z}^{2} \backslash\{(0,0)\}} \frac{\sqrt{\Delta(h)}}{|h(p, q)|}
$$

of Diophantine approximations of real, indefinite, binary quadratic forms $h(x, y)=a x^{2}+b x y+c y^{2}$ with positive discriminant $\Delta(h)=b^{2}-4 a c>0$. In this context, Markov proved that

$$
L \cap[\sqrt{5}, 3)=M \cap[\sqrt{5}, 3)=\left\{\sqrt{9-\frac{4}{z_{n}^{2}}}: n \in \mathbb{N}\right\},
$$

where $z_{n}$ is a Markov number, i.e., the largest coordinate of a solution $\left(x_{n}, y_{n}, z_{n}\right) \in \mathbb{N}^{3}$ of the Markov-Hurwitz equation

$$
x_{n}^{2}+y_{n}^{2}+z_{n}^{2}=3 x_{n} y_{n} z_{n} .
$$

2.1.1. Fermat's descent on Markov's cubic. The MarkovHurwitz equation determines a cubic surface $S$ whose integral points are called Markov triples. Since the MarkovHurwitz equation is quadratic on a given variable (when we freeze the other two variables), the cubic surface $S$ has a rich group of automorphisms made available by swapping roots of those quadratic equations: besides permuting the coordinates, we can replace $(x, y, z)$ by $(3 y z-x, y, z)$, $(x, 3 x z-y, z)$, or $(x, y, 3 x y-z)$ without leaving $S$. The last three automorphisms are called Vieta involutions and they were used by Markov to produce a descent argument showing that any Markov triple $(x, y, z) \in \mathbb{N}^{3}$ can be obtained

${ }^{2}$ It is possible to show that $\ell(\alpha)=\infty$ for Lebesgue almost every $\alpha$. Hence, the Lagrange spectrum tries to encode Diophantine properties of irrational numbers beyond the probabilistic dominant regime. 


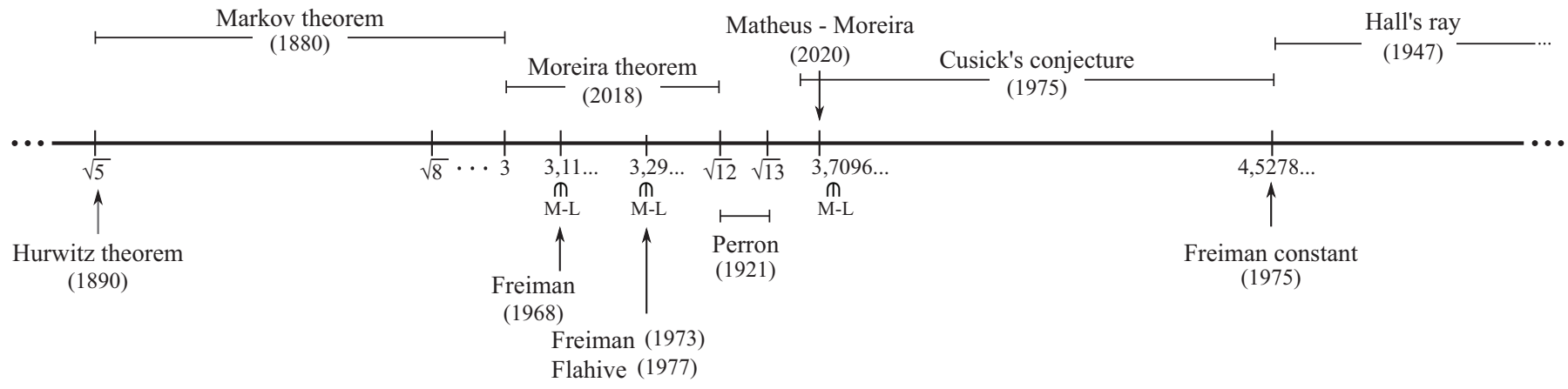

Figure 1.

from the fundamental solution $(1,1,1)$ after applying a sequence of permutations of coordinates and Vieta involutions.

In fact, it is not hard to see that a Markov triple $(x, y, z) \in \mathbb{N}^{3}$ with $x \leq y \leq z$ falls into two categories: either $x=y$ or $x<y<z$. In the first case, it turns out that $(x, y, z)=(1,1,1)$ or $(1,1,2)$. In the second case, applying the Vieta involution $(x, y, z) \mapsto\left(x, y, z^{\prime}\right)$ with $z^{\prime}=3 x y-z$ yields a Markov triple $\left(x, y, z^{\prime}\right)$, where $y$ is now the largest number. (Details are left to the reader.) By permuting the coordinates and repeating this argument finitely many times, we see that a sequence of Vieta involutions and permutations of coordinates allows us to convert the Markov triple $(x, y, z)$ into $(1,1,1)$, as desired.

2.1.2. The Markov tree. The descent argument above permits us to organize all ordered Markov triples $(x, y, z) \in \mathbb{N}^{3}$, $x \leq y \leq z$, into the so-called Markov tree whose branches connect ordered Markov triples deduced from each other by a Vieta involution (up to permutation of coordinates).

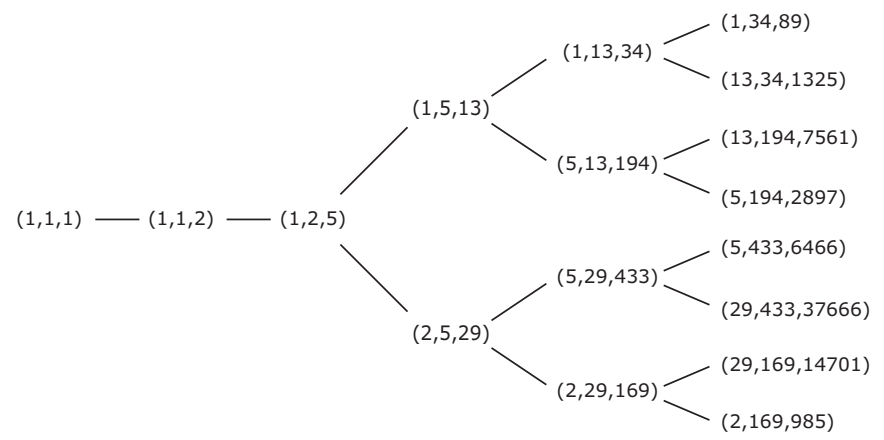

The knowledge of Markov's tree permits us to write down the first few elements of $M \cap[\sqrt{5}, 3)$ : since the first few Markov triples are $(1,1,1),(1,1,2),(1,2,5),(1,5,13)$, and $(2,5,29)$, we have that the first few Markov numbers ${ }^{3}$

\footnotetext{
${ }^{3}$ The attentive reader certainly noticed that some of these numbers are part of Fibonacci's sequence $\left(F_{n}\right)_{n \in \mathbb{N}}$ and this is not a coincidence: it is possible to check that $\left(1, F_{2 m-1}, F_{2 m+1}\right)$ is a Markov triple for all $m \in \mathbb{N}$.
}

are $1,2,5,13$, and 29 , so that

$$
M \cap[\sqrt{5}, 3)=\left\{\sqrt{5}, \sqrt{8}, \frac{\sqrt{221}}{5}, \frac{\sqrt{1517}}{13}, \frac{\sqrt{7565}}{29}, \ldots\right\} .
$$

2.1.3. Beyond the Markov tree.... The Markov tree and numbers are fascinating objects. For instance, it was conjectured by Frobenius in 1913 that Markov triples $(x, y, z) \in \mathbb{N}^{3}, x \leq y \leq z$, are actually determined by the Markov number $z$ (cf. Bombieri's survey article [Bom07])

Also, Zagier [Zag82] showed that the number $M(x)$ of Markov numbers below $x$ is

$$
M(x)=c(\log x)^{2}+O\left(\log x(\log \log x)^{2}\right),
$$

where $c=0.180717104711507 \ldots$ is an explicit constant, and, more recently, Baragar [Bar94] and Gamburd-MageeRonan [GMR19] studied the general problem of counting integral points on the Markov-Hurwitz varieties of the form

$$
x_{1}^{2}+\cdots+x_{n}^{2}=a x_{1} \cdots x_{n}+k,
$$

where $n \geq 3, a \geq 1$, and $k$ are integers.

Moreover, the Markov triples are related to lengths of simple closed geodesics on a certain hyperbolic oncepunctured torus: in fact, the commutator subgroup $\Gamma$ of $S L(2, \mathbb{Z})$ is an index 12 subgroup generated by $A_{0}=$ $\left(\begin{array}{cc}1 & -1 \\ -1 & 2\end{array}\right)$ and $B_{0}=\left(\begin{array}{ll}1 & 1 \\ 1 & 2\end{array}\right)$; the quotient $S L(2, \mathbb{R}) / \Gamma$ is the unit cotangent bundle of a hyperbolic once-punctured torus whose simple closed geodesics correspond to the elements $A \in \Gamma$ in a pair $(A, B)$ of generators of $\Gamma$; the hyperbolic lengths of these geodesics have the form $2 \cosh ^{-1}(\operatorname{tr}(A) / 2)$, so that they are related to Markov triples because Fricke proved that any generating pair $(A, B)$ of $\Gamma$ satisfies

$$
\operatorname{tr}(A)^{2}+\operatorname{tr}(B)^{2}+\operatorname{tr}(A B)^{2}=\operatorname{tr}(A) \operatorname{tr}(B) \operatorname{tr}(A B),
$$

i.e., $(\operatorname{tr}(A) / 3, \operatorname{tr}(B) / 3, \operatorname{tr}(A B) / 3)$ is a Markov triple.

Furthermore, it is known (cf. [Gol03]) that the level sets of the function $x(x, y, z)=x^{2}+y^{2}+z^{2}-x y z-2$ parametrize the elements of the $S L(2, \mathbb{R})$-character variety ${ }^{4}$ of

\footnotetext{
${ }^{4}$ Naively speaking, the G-character variety of a topological surface $S_{g, n}$ of genus $g$ with $n$ punctures is the set of equivalence classes of representations $p$ : $\pi_{1}\left(S_{g, n}\right) \rightarrow G$ modulo the natural action of $G$ by conjugation.
} 
once-punctured torii and each Markov triple $(x, y, z)$ produces an integral point $(3 x, 3 y, 3 z)$ of $x^{-1}(-2)$.

Finally, Bourgain-Gamburd-Sarnak [BGS16] investigated the family of graphs $\left(\mathcal{G}_{p}\right)$ (indexed by the set of prime numbers $p$ ) obtained by applying Vieta involutions and permutation of coordinates to the solutions in $\mathbb{F}_{p}^{3} \backslash\{(0,0,0)\}$ to the Markov-Hurwitz equation $x^{2}+y^{2}+z^{2}=3 x y z$. In this setting, they showed $\mathcal{G}_{p}$ has a giant component $\mathcal{C}_{p}$ in the sense that $\#\left(\mathcal{G}_{p} \backslash \mathcal{C}_{p}\right)=O_{\varepsilon}\left(p^{\varepsilon}\right)$ for all $\varepsilon>0$, and they used the technology involved in the proof of this statement to establish that almost all Markov numbers are composite, i.e.,

$$
\frac{\#\{p \text { prime Markov number } \leq T\}}{\#\{z \text { Markov number } \leq T\}} \rightarrow 0
$$

as $T \rightarrow \infty$. Also, they conjectured that the graphs $\mathcal{G}_{p}$ are connected $^{5}$ and they form an expander family. ${ }^{6}$

2.2. Continued fractions. The definition of the Lagrange spectrum suggests that we can study $L$ provided there is a method to find the best rational approximations of a given irrational number $\alpha$ (such as $22 / 7$ and $355 / 113$ for $\pi$ ).

As it turns out, one can guess the best rational approximations for $\alpha$ out of its continued fraction expansion. More precisely, given an irrational number $\alpha_{0}=\alpha$, let $a_{0}=\lfloor\alpha\rfloor$, so that $\alpha_{0}-a_{0} \in(0,1)$. We define recursively $\alpha_{n}=\frac{1}{\alpha_{n-1}-a_{n-1}}$ and $a_{n}=\left\lfloor\alpha_{n}\right\rfloor \in \mathbb{N}^{*}$ for all $n \in \mathbb{N}^{*}$. In this context, we say that $\alpha$ has continued fraction expansion

$$
\alpha=a_{0}+\frac{1}{a_{1}+\frac{1}{a_{2}+\frac{1}{\ddots}}}=\left[a_{0} ; a_{1}, a_{2}, \ldots\right]
$$

and we denote by

$$
\frac{p_{n}}{q_{n}}=a_{0}+\frac{1}{a_{1}+\frac{1}{\ddots+\frac{1}{a_{n}}}}=\left[a_{0} ; a_{1}, \ldots, a_{n}\right]
$$

the convergents of $\alpha$. For example, $\pi=[3 ; 7,15,1,292,1,1$, $1,2,1,3,1,14,2,1, \ldots]$, so that

$$
\frac{p_{1}}{q_{1}}=\frac{22}{7} \text { and } \quad \frac{p_{3}}{q_{3}}=\frac{355}{113} .
$$

It is possible to prove that $\left(p_{n} / q_{n}\right)$ provides the best rational approximations to $\alpha$ in the sense that every convergent $p_{n} / q_{n}$ is within $1 / q_{n}^{2}$ of $\alpha$, every convergent is closer to $\alpha$ than any rational number with smaller denominator, and every rational approximation $p / q$ that is within $1 / 2 q^{2}$ of $\alpha$ is a convergent.

In particular, the best constant

$$
\ell(\alpha)=\limsup _{p, q \rightarrow \infty} \frac{1}{|q(q \alpha-p)|}
$$

\footnotetext{
${ }^{5}$ The connectedness of $\mathcal{G}_{p}$ for all large $p$ was very recently established by Chen; cf. the arxiv preprint arXiv:2011.12940.

${ }^{6}$ That is, there is a uniform spectral gap for the adjacency matrices of these graphs.
}

of Diophantine approximation for $\alpha$ depends only on its convergents, i.e.,

$$
\ell(\alpha)=\limsup _{n \rightarrow \infty} \frac{1}{\left|q_{n}\left(q_{n} \alpha-p_{n}\right)\right|} .
$$

2.3. Perron's definition of the spectra. The basic formula

$$
\frac{1}{q_{n}\left(q_{n} \alpha-p_{n}\right)}=(-1)^{n}\left(\alpha_{n+1}+\beta_{n+1}\right)
$$

where $\beta_{n+1}:=q_{n-1} / q_{n}=\left[0 ; a_{n}, \ldots, a_{1}\right]$, led Perron to propose in 1921 the following dynamical interpretation of $L$. Let $\Sigma=\left(\mathbb{N}^{*}\right)^{\mathbb{Z}}$ be the (noncompact) symbolic space of biinfinite sequences of nonzero natural numbers. The left shift map $\sigma: \Sigma \rightarrow \Sigma$ is the dynamical system given by

$$
\sigma\left(\left(a_{n}\right)_{n \in \mathbb{Z}}\right)=\left(a_{n+1}\right)_{n \in \mathbb{Z}} .
$$

In this language, the Lagrange spectrum is the set of finite asymptotic records of heights of the orbits of $\sigma$ with respect to the (proper) height function $f: \Sigma \rightarrow \mathbb{R}, f\left(\left(a_{n}\right)_{n \in \mathbb{Z}}\right):=$ $\left[a_{0} ; a_{1}, a_{2}, \ldots\right]+\left[0 ; a_{-1}, a_{-2}, \ldots\right]$, i.e.,

$$
L=\left\{\limsup _{n \rightarrow \infty} f\left(\sigma^{n}(x)\right)<\infty: x \in \Sigma\right\} .
$$

To see this, embed a continued fraction in $\Sigma$ by filling in to the left of the 0 position with any sequence of nonzero natural numbers. Asymptotically these numbers do not contribute to the height as they are shifted to the left.

Interestingly enough, one can use the classical reduction theory of binary quadratic forms (due to Lagrange and Gauss) to prove that the Markov spectrum is the set of finite absolute records of heights of the orbits of $\sigma$ with respect to $f$, i.e.,

$$
M=\left\{\sup _{n \in \mathbb{Z}} f\left(\sigma^{n}(x)\right)<\infty: x \in \Sigma\right\} .
$$

From these dynamical characterizations of $L$ and $M$, Perron deduced that

- $\sup _{n \in \mathbb{Z}} f\left(\sigma^{n}(x)\right) \leq \sqrt{12}$ if and only if $x \in\{1,2\}^{\mathbb{Z}}$;

- $\sqrt{12}, \sqrt{13}, \frac{9 \sqrt{3}+65}{22} \in L$;

- $M \cap(\sqrt{12}, \sqrt{13})=M \cap\left(\sqrt{13}, \frac{9 \sqrt{3}+65}{22}\right)=\emptyset$.

Moreover, one can use this dynamical point of view to prove that

$$
L=\overline{\left\{\sup _{n \rightarrow \infty} f\left(\sigma^{n}(y)\right): y \in \Sigma \text { is periodic }\right\}}
$$

and

$$
M=\overline{\left\{\sup _{n \in \mathbb{Z}} f\left(\sigma^{n}(z)\right): z \in \Sigma \text { is eventually periodic }\right\} .}
$$

Here eventually periodic means eventually periodic on both sides (perhaps with different periods). Thus, $L \subset M$ are closed subsets of the real line. 
2.4. Dynamics on the modular surface. The shift map $\sigma: \Sigma \rightarrow \Sigma$ can be thought of as an invertible map extending the Gauss map $G:(0,1] \rightarrow[0,1), G(x)=\{1 / x\}$. Indeed, the definitions imply that the Gauss map acts on continued fraction expansions by left-shift on half-infinite sequences of natural numbers:

$$
G\left(\left[0 ; a_{1}, a_{2}, \ldots\right]\right)=\left[0 ; a_{2}, \ldots\right] .
$$

Using the well-known link (due to Artin, Cohn, Series, Arnoux, ...) between the Gauss map and the geodesic flow $g_{t}$ on the unit cotangent bundle $S L(2, \mathbb{R}) / S L(2, \mathbb{Z})$ to the modular surface (cf. [Arn94]), one can also describe the Lagrange spectrum as the set of finite asymptotic records of the heights of the orbits of a continuous-time, smooth dynamical system, namely,

$$
L=\left\{\limsup _{t \rightarrow \infty} H\left(g_{t}(x)\right)<\infty: x \in S L(2, \mathbb{R}) / S L(2, \mathbb{Z})\right\},
$$

where $H: S L(2, \mathbb{R}) / S L(2, \mathbb{Z}) \rightarrow \mathbb{R}$ is a certain (proper) function. ${ }^{7}$

2.5. The end of the classical spectra. The expression of the height function $f: \Sigma \rightarrow \mathbb{R}$ in Perron's definition of the spectra suggests that $L$ and $M$ are related to arithmetic sums of Cantor sets of real numbers whose continued fraction expansions have restricted digits.

In other terms, the study of projections of products of certain Cantor sets under the function $\pi: \mathbb{R}^{2} \rightarrow \mathbb{R}$, $\pi(x, y):=x+y$, should provide some insights into the fine structures of $L$ and $M$.

This idea was explored by Hall in 1947 to show that $L$ contains the half-line $[6, \infty)$. For this sake, Hall considered the continued fraction Cantor set $C(4)=\left\{\left[0 ; a_{1}, a_{2}, \ldots\right]\right.$ : $\left.1 \leq a_{i} \leq 4 \forall i \in \mathbb{N}\right\}$ and he established that

$$
\begin{aligned}
C(4)+C(4) & :=\{x+y:(x, y) \in C(4) \times C(4)\} \\
& =[\sqrt{2}-1,4(\sqrt{2}-1)]
\end{aligned}
$$

is an interval of length $>1$. This fact implies that given $\ell \in[6, \infty)$, one can find $c_{0} \in \mathbb{N}$ such that $5 \leq c_{0} \leq \ell$ and $\ell-c_{0} \in C(4)+C(4)$, say

$$
\ell=c_{0}+\left[0 ; a_{1}, a_{2}, \ldots\right]+\left[0 ; b_{1}, b_{2}, \ldots\right]
$$

with $1 \leq a_{i}, b_{i} \leq 4$ for all $i \in \mathbb{N}$. Thus, the irrational number $\alpha$ with continued fraction expansion

$$
\alpha=[0 ; \underbrace{b_{1}, c_{0}, a_{1}}_{1 \text { st block }}, \ldots, \underbrace{b_{n}, \ldots, b_{1}, c_{0}, a_{1}, \ldots, a_{n}}_{n \text {th block }}, \ldots]
$$

satisfies $\ell=\ell(\alpha) \in L$. (Consider successive leftward shifts that center the occurrences of $c_{0}$ at the 0 position.) Since $\ell \geq 6$ was arbitrary, we conclude that $L \supset[6, \infty)$.

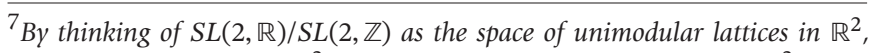
one has $H(x)=2 /$ sys $(x)^{2}$, where sys $(x)$ is the systole of $x \simeq g \mathbb{Z}^{2}, g \in$ $S L(2, \mathbb{R})$.
The largest half-line of the form $\left[c_{F}, \infty\right)$ included in $L$ is called Hall's ray. In 1975, Freiman famously claimed that

$$
c_{F}=4+\frac{253589820+283798 \sqrt{462}}{491993569}=4.527829566 \ldots
$$

2.6. Intermediate portions of $L$ and $M$. We saw above that $L \subset M$ are closed subsets of the real line such that $L \cap(-\infty, 3)=M \cap(-\infty, 3)=\{\sqrt{5}, \sqrt{8}, \ldots\}, L \cap\left[c_{F}, \infty\right)=$ $M \cap\left[c_{F}, \infty\right)=\left[c_{F}, \infty\right)$, and $\sqrt{12}, \sqrt{13} \in L$, but $(\sqrt{12}, \sqrt{13}) \cap$ $M=\emptyset$.

In particular, $L$ and $M$ coincide in the portions $(-\infty, 3)$, $[\sqrt{12}, \sqrt{13}]$, and $\left[c_{F}, \infty\right)$. Nonetheless, it was discovered by Freiman in 1968 that $M \backslash L \neq \emptyset$ : more concretely, Freiman found a countable subset of $M \backslash L$ located near 3.11.

Subsequently, Freiman discovered (in 1973) a new element of $M \backslash L$ near 3.29, and Flahive showed ${ }^{8}$ in 1977 that this element is the limit of an explicit sequence of elements of $M \backslash L$ near 3.29.

This "concentration" of examples of elements of $M \backslash L$ between 3 and 3.3 led Cusick to conjecture in 1975 that $L$ and $M$ should coincide above $\sqrt{12}$.

In any case, the previous paragraphs hint that the intermediate portions of the spectra (between 3 and $c_{F}$ ) might have a complicated structure in comparison to their beginning and ending. Indeed, as we will see, these intermediate portions of the spectra have a quite interesting fractal structure.

In order to state the next results, we will recall the notion of Hausdorff dimension of a subset $X$ of a Euclidean space (which can be easily extended to a general metric space): it measures how hard it is to efficiently cover $X$ by balls: given $X \subset \mathbb{R}^{m}$, we define

$$
\operatorname{dim}(X)=\inf \left\{s>0: \inf _{X \subset \bigcup_{n \in \mathbb{N}} B\left(x_{n}, r_{n}\right)} \sum_{n \in \mathbb{N}} r_{n}^{s}=0\right\} .
$$

We always have $0 \leq \operatorname{dim}(X) \leq m$. A countable set has zero Hausdorff dimension and any set $X \subset \mathbb{R}^{m}$ with $\operatorname{dim}(X)<$ $m$ has zero $m$-dimensional Lebesgue measure.

In 1971, Hall gave an upper bound on the fractal complexity of $M \cap[\sqrt{5}, \sqrt{10}]$. More precisely, he used Perron's definition of the spectra to establish that $M \cap[3, \sqrt{10}] \subset$ $2+U+U=\{2+u+v: u, v \in U\}$, where

$$
U=\left\{\left[0 ; a_{1}, a_{2}, \ldots\right]:\left(a_{i} a_{i+1} a_{i+2}\right) \neq(121) \forall i \in \mathbb{N}^{*}\right\} .
$$

After that, he analyzed the sizes of all intervals covering $U$ with extremities of the form $\left[0 ; a_{1}, \ldots, a_{n}, \overline{2122}\right]$ and $\left[0 ; a_{1}, \ldots, a_{n}, \overline{1222}\right]$ (where $\bar{w}$ stands for the periodic sequence obtained by infinite concatenation of a string $w$ )

\footnotetext{
${ }^{8}$ Actually, in his unpublished PhD thesis from 1976, Y.-C. You found an uncountable subset of $M \backslash L$ near 3.29 which is bi-Lipschitz homeomorphic to the Cantor set of continued fraction expansions obtained by concatenations of the words 11 and 22 .
} 
in order to show that the Hausdorff dimension of $U$ is at most 0.465 . From this estimate, it is possible to infer that the Hausdorff dimension of $U+U=\pi(U \times U)$ has Hausdorff dimension at most $2 \cdot \operatorname{dim}(U)<0.93$ and, a fortiori, $M \cap[\sqrt{5}, \sqrt{10}] \subset 2+U+U$ has zero Lebesgue measure.

On the other hand, it is believed that $L$ and $M$ should contain nontrivial intervals before $c_{F}$ : for instance, a folklore question (appearing on page 71 of Cusick-Flahive's book [CF89]) asks whether $L \cap[\sqrt{5}, \sqrt{12}]$ has nonempty interior, and Berstein conjectured in 1973 that $[4.1,4.52] \subset L$. Here, it is worth pointing out that the inspiration for the first (folklore) question comes from:

- Perron's result that $M \cap[\sqrt{5}, \sqrt{12}]$ is closely related to the arithmetic sum $C(2)+C(2)$, where $C(2):=$ $\left\{\left[0 ; a_{1}, a_{2}, \ldots\right]: 1 \leq a_{i} \leq 2 \forall i\right\}$;

- the expectation ${ }^{9}$ that $C(2)+C(2)$ contains intervals because $C(2)+C(2)$ is the projection $\pi(C(2) \times C(2))$ of a planar "nonlinear" Cantor set $C(2) \times C(2) \subset$ $\mathbb{R}^{2}$ with Hausdorff dimension $2 \cdot \operatorname{dim}(C(2))>1$.

Also, Berstein thinks that $[4.1,4.52] \subset L$ because of Freiman's work on the computation of the beginning $c_{F}$ of Hall's ray.

2.7. Recent results about $M \cap\left(3, c_{F}\right)$. Despite the strong belief (expressed by the conjectures and questions in the previous subsection) that $M \cap\left(3, c_{F}\right)$ must have an intricate structure, the first rigorous result in this direction was obtained only in 2018 by the second author [Mor18]. In fact, he showed that:

- for each $t \in \mathbb{R}$, the Hausdorff dimension $d(t)$ of $L \cap(-\infty, t)$ coincides with the Hausdorff dimension of $M \cap(-\infty, t)$, i.e., $M \backslash L$ is not big enough to create jumps in dimension between $L$ and $M$;

- $d(t)$ is a continuous, non-Hölder function of $t$ such that $d(3+\varepsilon)>0$ for all $\varepsilon>0$ and $d(\sqrt{12})=1$.

The second item above implies that $L$ and $M$ necessarily contain complicated fractal sets. For instance, it is not difficult to check that if $K \subset \mathbb{R}$ is a Cantor set defined by simple interactive (dynamical) rules like Cantor's middlethird set, then the function $t \mapsto \operatorname{dim}(K \cap(-\infty, t))$ is always piecewise constant and discontinuous:

$$
\operatorname{dim}(K \cap(-\infty, t))=\left\{\begin{array}{cc}
0 & \text { if } t \leq \min K \\
\operatorname{dim}(K) & \text { if } t>\min K
\end{array}\right.
$$

More recently, we investigated in [MM19a], [MM19b], and [MM20] the fine structure of $M \backslash L$ and we proved that it is richer than conjectured by Cusick. More precisely,

\footnotetext{
${ }^{9}$ The typical projections of generic planar Cantor sets with Hausdorff dimension $>1$ are expected to contain intervals thanks to the combination of Marstrand's theorem asserting that the projections in almost all directions of a subset of $\mathbb{R}^{2}$ with Hausdorff dimension $>1$ have positive Lebesgue measures and the work of Yoccoz and the second author on the stable intersections of Cantor sets via renormalization techniques.
}

there are three open intervals $I_{1}, I_{2}$, and $I_{3}$ near $3.11,3.29$, and 3.7 such that:

- the sizes of $I_{1}, I_{2}, I_{3}$ are $\sim 2 \cdot 10^{-10}, 2 \cdot 10^{-7}, 10^{-10}$;

- the extremities of $I_{j}$ belong to $L$, but $L \cap I_{j}=\emptyset$ for each $1 \leq j \leq 3$;

- $(M \backslash L) \cap I_{j}, 1 \leq j \leq 3$, are closed subsets with Hausdorff dimensions $>0.26,0.353,0.531$, resp.;

- $I_{1}$ and $I_{2}$ contain the examples of elements of $M \backslash L$ previously found by Freiman and Flahive, and the elements of $(M \backslash L) \cap I_{3}$ provide a negative answer to Cusick's conjecture that $L$ and $M$ should coincide above $\sqrt{12}$.

On the other hand, we proved that $M \backslash L$ is not very rich because $\operatorname{dim}(M \backslash L)<0.987$.

Besides the metric results discussed above, our techniques also give topological consequences for $L$ and $M$ : for example, the subset $L^{\prime}$, defined to be the set of nonisolated points of $L$, is perfect, i.e., $L^{\prime}=L^{\prime \prime}$, and the interiors of $L$ and $M$ coincide. However, we ignore whether $M^{\prime}$ is a perfect set, and, contrary to the initial impression given by the fact that $(M \backslash L) \cap I_{j}, 1 \leq j \leq 3$, are closed subsets, Lima, Vieira, and the authors proved that $M \backslash L$ is not closed.

In an attempt to further investigate interesting questions about the structure of $M \cap\left(3, c_{F}\right)$, Delecroix and the authors [DMM20] developed an algorithm providing $1 / Q$ approximations to $L$ and $M$ after a running time $O\left(Q^{2.367}\right)$. This algorithm was implemented (on Sage) by Delecroix to produce Figure 2 of $L_{2}:=L \cap[\sqrt{5}, \sqrt{12}]$, but unfortunately, we could not use this algorithm yet to get definite ideas about Berstein's conjecture that $[4.1,4.52] \subset L$. Nevertheless, we hope that some variant of this algorithm will be helpful in the future because its running time is not very big in comparison with the "naive" algorithm stemming from the characterizations of $L$ and $M$ via the closures of the values of height records of periodic and eventually periodic elements of $\Sigma=\left(\mathbb{N}^{*}\right)^{\mathbb{Z}}$.

The common theme behind all results described in this subsection is the study of portions of $L$ and $M$ via the fractal geometry of certain types of dynamically defined Cantor sets. In the next section, we will briefly discuss these objects and we will make some comments about their applications to the proofs of our results on the Hausdorff dimensions of $L \cap(-\infty, t), M \cap(-\infty, t)$, and $M \backslash L$.

\section{Dynamical Cantor Sets}

Dynamically defined (or regular) Cantor sets on the line are one-dimensional hyperbolic sets, defined by expanding maps and have some kind of self-similarity property: small parts of them are diffeomorphic to big parts with uniformly bounded distortion. 


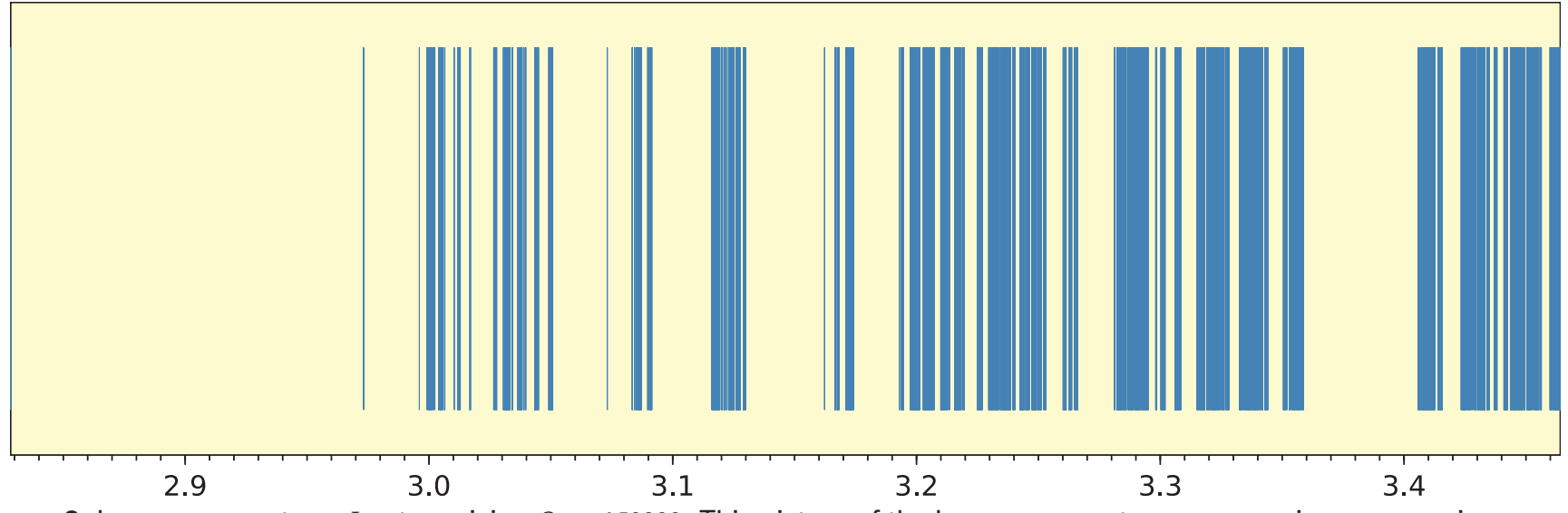

Figure 2. Lagrange spectrum $L_{2}$ at precision $Q_{2}=150000$. This picture of the Lagrange spectrum appears in our paper in collaboration with Vincent Delecroix, "Approximations of the Lagrange and Markov spectra," Math. Comp. 89 (2020), $2521-2536$. We would like to thank Vincent who kindly allowed us to reproduce it here.

A dynamically defined Cantor set $K \subset R$ is

$$
K=\bigcap_{n \in \mathbb{N}} \psi^{-n}\left(I_{1} \cup \cdots \cup I_{k}\right)
$$

where $\psi: I_{1} \cup \cdots \cup I_{k} \rightarrow I$ is a smooth (at least $C^{1+\alpha}$ for some $\alpha>0$ ) map from a collection of disjoint compact intervals $I_{1}, \ldots, I_{k}$ onto the convex hull $I$ of their union such that:

- $\psi$ is expanding: $\left|\psi^{\prime}(x)\right|>1 \forall x \in I_{1} \cup \cdots \cup I_{k}$;

- $\left\{I_{1}, \ldots, I_{k}\right\}$ is a Markov partition: each $\psi\left(I_{i}\right)$ is the convex hull of the union of some of the intervals $I_{j}$;

- $\psi$ is topologically mixing: there exists $n_{0} \in \mathbb{N}$ with $\psi^{n_{0}}\left(K \cap I_{i}\right)=K$ for all $1 \leq i \leq k$.

The most famous example of a dynamically defined Cantor set is arguably Cantor's middle-third set

$$
K_{1 / 3}=\left\{\sum_{n=1}^{\infty} \frac{a_{n}}{3^{n}}:\left(a_{n}\right)_{n \in \mathbb{N}} \in\{0,2\}^{\mathbb{N}}\right\}
$$

obtained by dividing $[0,1]$ into three intervals with equal lengths, removing the middle one $(1 / 3,2 / 3)$, dividing the remaining intervals into three equal subintervals, removing the middle ones, repeating this procedure ad infinitum, and keeping only the points of $[0,1]$ never falling into an excluded subinterval.

In fact, $K_{1 / 3}=\bigcap_{n \in \mathbb{N}} \phi^{-n}\left(I_{1} \cup I_{2}\right)$, where $I_{1}=[0,1 / 3]$, $I_{2}=[2 / 3,1]$, and

$$
\phi(x)=\left\{\begin{array}{cc}
3 x & \text { if } x \in I_{1} \\
3 x-2 & \text { if } x \in I_{2}
\end{array}\right.
$$

The geometry of dynamical Cantor sets was studied in depth by several authors over the last 100 years. In particular, we dispose nowadays of several methods to compute the Hausdorff dimension of dynamical Cantor sets. For instance, given a dynamical Cantor set $K$ generated by $\psi: I_{1} \cup \cdots \cup I_{k} \rightarrow I$, for each $m \in \mathbb{N}$, we can consider the collection $\mathcal{R}_{m}$ of intervals consisting of the connected components of $\bigcap_{n=1}^{m} \psi^{-n}\left(I_{1} \cup \cdots \cup I_{k}\right)$. By definition, $\mathcal{R}_{m}$ is a cover of $K$ and it can be used to compute $\operatorname{dim}(K)$ : if we denote by $\lambda(J)=\left.\min \left|\psi^{\prime}\right|_{J}|, \Lambda(J)=\max | \psi^{\prime}\right|_{J} \mid$ and we fix a mixing time $n_{0}$ for $\psi$ (i.e., $\psi^{n_{0}}\left(I_{j} \cap K\right)=K$ for each $1 \leq j \leq k$ ), then it is possible to prove that $\alpha_{m} \leq \operatorname{dim}(K) \leq \operatorname{dim}(K) \leq \beta_{m}$, where

$$
\sum_{J \in \mathcal{R}_{m}} \frac{1}{\lambda(J)^{\beta_{m}}}=1 \text { and } \sum_{J \in \mathcal{R}_{m}} \frac{1}{\Lambda(J)^{\alpha_{m}}}=\max \left|\left(\psi^{n_{0}-1}\right)^{\prime}\right|
$$

(cf. pages 68 to 70 of Palis-Takens' book [PT93]). These bounds allow an exact calculation of the Hausdorff dimension of dynamical Cantor sets associated to piecewise affine maps $\psi$ with full branches (i.e., mixing time $n_{0}=1$ ) such as Cantor's middle-third set $K_{1 / 3}$ : indeed, $K_{1 / 3}$ is defined by a map $\phi: I_{1} \cup I_{2} \rightarrow I$ such that $\phi^{\prime} \equiv 3$ (and $\left.n_{0}=1\right)$, so that $\alpha_{1} \leq \operatorname{dim}\left(K_{1 / 3}\right) \leq \beta_{1}$, where

$$
2(1 / 3)^{\alpha_{1}}=1=2(1 / 3)^{\beta_{1}},
$$

i.e., $\operatorname{dim}\left(K_{1 / 3}\right)=\log 2 / \log 3$.

Unfortunately, the elementary technique described in the previous paragraph doesn't permit us to compute the dimension of dynamical Cantor sets $K$ associated maps $\psi: I_{1} \cup \cdots \cup I_{k} \rightarrow I$ that are nonessentially affine, that is, there is no smooth change of coordinates $h: I \rightarrow J$ making $h \circ \psi \circ h^{-1}$ into a piecewise affine map. In fact, one can check that $\beta_{m}-\alpha_{m}=O(1 / m)$ in general, so that the convergence of $\alpha_{m}$ and $\beta_{m}$ to $\operatorname{dim}(K)$ might be quite slow in the nonessentially affine situations related to the classical spectra $L$ and $M$.

Nevertheless, Bowen [Bow79] discovered in 1979 a famous formula for the Hausdorff dimension of dynamical Cantor sets $K$ related to a family $\left(\mathcal{L}_{t}\right)_{t \in(0,1)}$ of 
Ruelle-Perron-Frobenius transfer operators

$$
\mathcal{L}_{t} f(x)=\sum_{y \in \psi^{-1}(x)} f(y)\left|\psi^{\prime}(y)\right|^{-t}
$$

acting on adequate spaces of smooth functions $f: I \rightarrow$ $\mathbb{R}$. This formula was subsequently explored by several authors (including Falk, Hensley, Jenkinson, McMullen, Nussbaum, Pollicott, Vytnova) for a fast computation of several digits of $\operatorname{dim}(K)$.

In particular, this method was successfully explored by Jenkinson and Pollicott in 2018 to compute the first 100 decimal digits of the Hausdorff dimension $\operatorname{dim}(C(2))$ of the Cantor set $C(2)=\left\{\left[0 ; a_{1}, a_{2}, \ldots\right]: 1 \leq a_{i} \leq 2 \forall i\right\}$ which is dynamically defined by the piecewise real-analytic map given by the restriction of the Gauss map to the intervals $[[0 ; 2 \overline{12}],[0 ; 2, \overline{21}]]$ and $[[0 ; 1 \overline{12}],[0 ; 1 \overline{21}]]$. An outcome of their calculations is that

$$
\operatorname{dim}(C(2))=0.531280506277205141624468647368 \ldots .
$$

3.1. Dimension of Gauss-Cantor sets. For our purpose of studying the classical spectra, the relevant class of dynamical Cantor sets are the so-called Gauss-Cantor sets defined as follows.

Let $B \subset \bigcup_{n \geq 1}\left(\mathbb{N}^{*}\right)^{n}$ be a finite set of finite words which is primitive in the sense that none of its elements is a prefix of another one. The corresponding complete Gauss-Cantor set is

$$
K(B)=\left\{\left[0 ; \beta_{1}, \beta_{2}, \ldots\right]: \beta_{i} \in B \forall i \geq 1\right\} .
$$

The simplest examples ${ }^{10}$ of complete Gauss-Cantor sets are the sets

$$
C(k):=\left\{\left[0 ; a_{1}, a_{2}, \ldots\right]: 1 \leq a_{i} \leq k \forall i\right\}
$$

for $k \geq 2$.

In general, a Gauss-Cantor set is a set of the type

$$
K(\gamma, B)=\left\{\left[0 ; \gamma, \beta_{1}, \beta_{2}, \ldots\right]: \beta_{i} \in B \forall i \geq 1\right\},
$$

where $\gamma \in \bigcup_{n \geq 1}\left(\mathbb{N}^{*}\right)^{n}$ is a finite word.

Notice that $K(\gamma, B)$ is the image of $K(B)$ under the biLipschitz homeomorphism

$$
[0 ; \gamma, x] \mapsto[0 ; x]=G^{|\gamma|}([0 ; \gamma, x]),
$$

where $G$ is the Gauss map and $|\gamma|$ is the size of $\gamma$. In particular, $K(B)$ and $K(\gamma, B)$ have the same Hausdorff dimension.

Complete Gauss-Cantor sets are dynamical Cantor sets defined by iterates of the Gauss map $G$. Indeed, $K(B)$ is a dynamical Cantor set $\psi: \bigcup_{\beta \in B} I(\beta) \rightarrow I$, where $\left.\psi\right|_{I(\beta)}=G^{|\beta|}$ and $I(\beta)$ are intervals with extremities of the form $\left[0 ; \beta, x_{\beta}\right]$ and $\left[0 ; \beta, y_{\beta}\right]$ for adequate choices of $x_{\beta}, y_{\beta} \in B^{\mathbb{N}}$.

Similarly, one can verify that, in general, Gauss-Cantor sets are also dynamical Cantor sets.

\footnotetext{
${ }^{10}$ Note that $C(4)$ and $C(2)$ already appeared in our discussions of the ending and the intermediate portions of the classical spectra $L$ and $M$.
}

It is not difficult to show that any Gauss-Cantor set is nonessentially affine, i.e., it is dynamically defined by a map $\psi: I_{1} \cup \cdots \cup I_{k} \rightarrow I$ such that there is no smooth change of coordinates $h$ making the second derivative of $h \circ \psi \circ h^{-1}$ vanish identically on $h(K)$. Thus, a Gauss-Cantor set is geometrically more intricate than dynamical Cantor sets given by piecewise affine maps (such as Cantor's middlethird set) and, hence, we do not expect to get an exact formula for its Hausdorff dimension (but only some high precision approximation coming from variants of Bowen's formula, for example).

Nonetheless, the second author discovered ${ }^{11}$ that the renormalization techniques (including the so-called scale recurrence lemma) introduced by Yoccoz and him [dAMY01] in their study of stable intersections of dynamical Cantor sets can be used to prove that if $K$ is a nonessentially affine dynamical Cantor set and $K^{\prime}$ is an arbitrary dynamical Cantor set, then the projection $\pi\left(K \times K^{\prime}\right)=$ $K+K^{\prime}$ of $K \times K^{\prime}$ under $\pi(x, y)=x+y$ has expected Hausdorff dimension

$$
\operatorname{dim}\left(K+K^{\prime}\right)=\min \left\{1, \operatorname{dim}(K)+\operatorname{dim}\left(K^{\prime}\right)\right\} .
$$

In particular, Gauss-Cantor sets satisfy the following dimension formula: for any finite words $\gamma, \gamma^{\prime}$ and finite sets of finite words $B, B^{\prime}$, one has

$$
\begin{gathered}
\operatorname{dim}\left(K(\gamma, B)+K\left(\gamma^{\prime}, B^{\prime}\right)\right) \\
=\min \left\{1, \operatorname{dim}(K(B))+\operatorname{dim}\left(K\left(B^{\prime}\right)\right)\right\} .
\end{gathered}
$$

Also, it is worth pointing out the following ${ }^{12}$ useful "symmetry" on the Hausdorff dimension of Gauss-Cantor sets. Given a finite set of finite words $B$, denote by $B^{T}=$ $\left\{\beta^{T}: \beta \in B\right\}$ the transpose of $B$, where $\beta^{T}:=\left(a_{n}, \ldots, a_{1}\right)$ stands as usual for the transpose of $\beta=\left(a_{1}, \ldots, a_{n}\right)$. Then, the Hausdorff dimension of the Gauss-Cantor sets associated to $B$ and $B^{T}$ are equal:

$$
\operatorname{dim}(K(B))=\operatorname{dim}\left(K\left(B^{T}\right)\right) .
$$

In a certain sense, the proof of this fact goes back to Euler: indeed, he proved that for any finite word $\beta$, if $[0 ; \beta]=$ $p_{n} / q_{n}$, then $\left[0 ; \beta^{T}\right]=r_{n} / q_{n}$. Since the lengths of the intervals $I\left(\beta_{1} \beta_{2} \cdots \beta_{k}\right)$ in the $k$ th step of the construction of $K(B)$ depend only on the denominators of the convergents of $\left[0 ; \beta_{1} \beta_{2} \cdots \beta_{k}\right]$, Euler's result says that $K(B)$ and $K\left(B^{T}\right)$ are Cantor sets constructed from small intervals with comparable lengths, and, a fortiori, they have the same Hausdorff dimension.

\footnotetext{
${ }^{11}$ A version of this formula was obtained by Hochman and Shmerkin.

${ }^{12}$ The dynamical explanation for this symmetry is the fact that the Gauss map has a smooth, area-preserving, natural extension.
} 
3.2. Dimension across the spectra. As we said at the end of $\$ 2.7$, we want to study portions of $L$ and $M$ via dynamical Cantor sets. In particular, a central idea towards the main theorem of [Mor18] about the continuity of the Hausdorff dimension across the classical spectra is to approximate $L \cap(-\infty, t)$ and $M \cap(-\infty, t)$ from inside and outside by arithmetic sums of Gauss-Cantor sets. Using this method, the second author proved that $\operatorname{dim}(L \cap(-\infty, t))$ and $\operatorname{dim}(M \cap(-\infty, t))$ are continuous functions of $t$.

In more detail, let $D(t)=\operatorname{dim}\left(\ell^{-1}(-\infty, t)\right)$ (where $\ell(\alpha)$ is the best constant ${ }^{13}$ of the Diophantine approximation of $\alpha$ ). Given $t$ such that $D(t)>0$ and $\epsilon>0$, the second author proved the existence of

- a parameter $\delta>0$,

- a finite set of positive integers $\left(a_{j}\right)_{1 \leq j \leq m+1}$,

- a finite set of finite prefixes $\left\{\gamma_{j}\right\}_{j=1}^{2 m+2} \subset \bigcup_{n \geq 1}\left(\mathbb{N}^{*}\right)^{n}$, and

- two finite sets $B, B^{\prime}$ of finite words

such that the translated arithmetic sum of Gauss-Cantor sets

$$
a_{m+1}+K\left(\gamma_{2 m+1}, B^{\prime}\right)+K\left(\gamma_{2 m+2},\left(B^{\prime}\right)^{T}\right)
$$

is contained in $L \cap(-\infty, t-\delta)$, the union of translated arithmetic sums of Gauss-Cantor sets

$$
\bigcup_{j \leq m}\left(a_{j}+K\left(\gamma_{2 j-1}, B\right)+K\left(\gamma_{2 j}, B^{T}\right)\right)
$$

contains $M \cap(-\infty, t+\delta)$, and

$$
D(t)-\epsilon<\operatorname{dim}\left(K\left(B^{\prime}\right)\right) \leq D(t) \leq \operatorname{dim}(K(B))<D(t)+\epsilon .
$$

In particular, these facts together with the dimension formula and Euler's symmetry imply that

$$
\begin{gathered}
\min \{1,2 D(t)-2 \epsilon\} \leq \min \left\{1,2 \operatorname{dim}\left(K\left(B^{\prime}\right)\right)\right\} \\
=\min \left\{1, \operatorname{dim}\left(K\left(B^{\prime}\right)\right)+\operatorname{dim}\left(K\left(\left(B^{\prime}\right)^{T}\right)\right)\right\} \\
\leq \operatorname{dim}(L \cap(-\infty, t-\delta)) \\
\leq \operatorname{dim}(L \cap(-\infty, t))
\end{gathered}
$$

and

$$
\begin{gathered}
\operatorname{dim}(M \cap(-\infty, t)) \leq \operatorname{dim}(M \cap(-\infty, t+\delta)) \\
\leq \min \left\{1, \operatorname{dim}(K(B))+\operatorname{dim}\left(K\left(B^{T}\right)\right)\right\} \\
=\min \{1,2 \operatorname{dim}(K(B))\} \\
\leq \min \{1,2 D(t)+2 \epsilon\} .
\end{gathered}
$$

Since $\epsilon>0$ was arbitrary, we conclude that

$$
\operatorname{dim}(L \cap(-\infty, t))=\operatorname{dim}(M \cap(-\infty, t))=\min \{1,2 D(t)\}
$$

is a continuous function of $t$.

In 1982, Bumby extended Hall's ideas in $\$ 2.6$ to give a computer-assisted argument indicating that $D(3.33437)<$ $1 / 2$ and $D(3.3344)>1 / 2$, so that

$$
3.33437<\inf \{t: \operatorname{dim}(M \cap[\sqrt{5}, t])=1\}<3.3344
$$

in view of our current discussion.

\footnotetext{
${ }^{13} \mathrm{Cf}$. the beginning of $\$ 2$.
}

3.3. Bounds on $\operatorname{dim}(M \backslash L)$. In [MM20], we explored the geometry of the intersections of the so-called local stable and unstable sets of the shift dynamics $\sigma$ to prove that $M \backslash L \supset a+K(\gamma, B)$, where $a=[3 ; 3, \overline{2,1,2,2,2,3,3}]$, $\gamma=(2,2,2,1,2,3,3,2,2,2,1,2,2,1,2,1,2,1,2)$, and $B=$ $\{1,2\}$. Thus, $\operatorname{dim}(M \backslash L) \geq \operatorname{dim}(K(\gamma, B))=\operatorname{dim}(K(B))=$ $\operatorname{dim}(C(2))>0.531$.

After giving this brief discussion of lower bounds on $\operatorname{dim}((M \backslash L)$, let us now discuss how to give nontrivial upper estimates for $\operatorname{dim}(M \backslash L)$ : we first chop $(3, \sqrt{21})$ into several small intervals $(\mu, \nu)$. The basic idea from [MM20] is that we can study $(M \backslash L) \cap(\mu, \nu)$ by taking two symmetric transitive subshifts of finite type $B \subset C \subset\left(\mathbb{N}^{*}\right)^{\mathbb{Z}}$ with $m(x)<\mu$ for all $x \in B$ and any $y \in\left(\mathbb{N}^{*}\right)^{\mathbb{Z}}$ with $m(y)<v$ belongs to $C$, and by noticing that a shadowing lemma type argument implies that, up to transposition, any sequence $z$ with $m(z) \in(M \backslash L) \cap(\mu, \nu)$ has the property that if $N$ is large, $n \geq N$, and $\tau, \tau^{\prime}$ are distinct finite strings such that $z_{-N} \cdots z_{n} \tau$ and $z_{-N} \cdots z_{n} \tau^{\prime}$ can be extended into two sequences $\cdots z_{-N} \cdots z_{n} \tau \alpha$ and $\cdots z_{-N} \cdots z_{n} \tau^{\prime} \alpha^{\prime}$ with Markov values in $(M \backslash L) \cap(\mu, \nu)$, then the unstable Cantor set $K(B)=\left\{\left[0 ; \theta_{1}, \theta_{2}, \ldots\right]:\left(\theta_{n}\right)_{n \in \mathbb{Z}} \in B\right\}$ of $B$ doesn't intersect the interval $\left[[0 ; \tau \alpha],\left[0 ; \tau^{\prime} \alpha^{\prime}\right]\right]$ : in other terms, the allowed continuations of $z$ with $m(z) \in(M \backslash L) \cap(\mu, \nu)$ live in a small Cantor set $K_{G}$ in the gaps of $K(B)$, so that $\operatorname{dim}((M \backslash L) \cap(\mu, \nu)) \leq \operatorname{dim}(K(C))+\operatorname{dim}\left(K_{G}\right)$.

For instance, for $(\mu, \nu)=(\sqrt{10}, \sqrt{12})$, we take $B=$ $\{11,22\}^{\mathbb{Z}}$ and $C=\{1,2\}^{\mathbb{Z}}$, and we show that, if $\tau \alpha$ starts with 1 and $\tau^{\prime} \alpha^{\prime}$ starts with 2 , then $\tau \alpha$ starts necessarily with 112 and $\tau^{\prime} \alpha^{\prime}$ starts necessarily with 221 . We use this to get the estimate $\operatorname{dim}\left(K_{G}\right)<0.174813$, so

$$
\begin{aligned}
\operatorname{dim}((M \backslash L) \cap[\sqrt{10}, \sqrt{12}]) & \\
& <0.531281+0.174813=0.706094 .
\end{aligned}
$$

\section{Beyond the Classical Spectra}

Partly inspired by Perron's characterization of the classical spectra, several authors (including Maucourant, Paulin, Parkkonen, and the second author) proposed dynamical generalizations of the Markov and Lagrange spectra. In a nutshell, dynamical Lagrange and Markov spectra are obtained after replacing $\sigma$ by a general dynamical system and $f$ by a general height function: for instance, given a homeomorphism $\varphi: M \rightarrow M$ of a topological space $M$, a compact $\varphi$-invariant subset $\Lambda$ of $M$, and a continuous function $f: M \rightarrow \mathbb{R}$, the dynamical Lagrange and Markov spectra associated to $(f, \Lambda)$ are

$$
L(f, \Lambda):=\left\{\limsup _{n \rightarrow \infty} f\left(\varphi^{n}(x)\right): x \in \Lambda\right\}
$$

and

$$
M(f, \Lambda)=\left\{\sup _{n \in \mathbb{Z}} f\left(\varphi^{n}(x)\right): x \in \Lambda\right\} .
$$


A direct generalization of the classical spectra is provided by the dynamical spectra associated to a diffeomorphism $\varphi: M \rightarrow M$ of a surface $M$ acting on a horseshoe ${ }^{14}$ and a typical differentiable real function $f$. In fact, arbitrarily large compact parts of the classical Markov and Lagrange spectra can be viewed as dynamical Markov and Lagrange spectra associated to horseshoes of conservative (i.e., area-preserving) diffeomorphisms. More precisely, as it is explained in [Arn94], for each $m \geq 2$, the map $T_{1}:(0,1) \times(0,1) \rightarrow[0,1) \times(0,1)$ given by

$$
T_{1}(x, y)=\left(\left\{\frac{1}{x}\right\}, \frac{1}{y+\lfloor 1 / x\rfloor}\right)
$$

preserves a smooth area-form near the horseshoe $\Lambda(m)=$ $C(m) \times C(m)$ corresponding to the maximal invariant set of $\left(\frac{1}{m+1}, 1\right) \times(0,1)$. In particular, since

$$
\begin{aligned}
& T_{1}\left(\left[0 ; a_{0}, a_{1}, a_{2}, \ldots\right],\left[0 ; b_{1}, b_{2}, b_{3}, \ldots\right]\right) \\
= & \left(\left[0 ; a_{1}, a_{2}, a_{3}, \ldots\right],\left[0 ; a_{0}, b_{1}, b_{2}, \ldots\right]\right),
\end{aligned}
$$

we see that $T_{1}$ is a (piecewise) smooth, conservative realization of the natural extension of the Gauss map (compare with (1)). The dynamical Markov and Lagrange spectra of $\left(T_{1}, \Lambda(m)\right)$ with respect to the function $f(x, y)=y+\frac{1}{x}$ have the same intersections with $(-\infty, m+1]$ as the classical Markov and Lagrange spectra.

In 2018, Cerqueira and the authors established the continuity of the Hausdorff dimension across the dynamical Lagrange and Markov spectra of typical thin horseshoes of conservative surface diffeomorphisms with respect to typical smooth functions (in analogy with the main continuity result in $\$ 2.7$ for $L$ and $M$ ). More precisely, let $\varphi_{0}$ be a smooth diffeomorphism of a surface $M^{2}$ preserving an area-form $\omega$. Suppose that $\varphi_{0}$ possesses a thin horseshoe $\Lambda_{0}$ in the sense that its Hausdorff dimension is $\operatorname{dim}\left(\Lambda_{0}\right)<1$. Denote by $\mathcal{U}$ a small $C^{\infty}$ neighborhood of $\varphi_{0}$ in the space Diff $\omega_{\omega}^{\infty}(M)$ of smooth area-preserving diffeomorphisms of $M$ such that $\Lambda_{0}$ admits a continuation ${ }^{15}$ $\Lambda$ for every $\varphi \in \mathcal{U}$. If $\mathcal{U} \subset \operatorname{Diff}_{\omega}^{\infty}(M)$ is sufficiently small, then there exists a Baire residual subset $\mathcal{U}^{* *} \subset \mathcal{U}$ with the following property. For every $\varphi \in \mathcal{U}^{* *}$ and $r \geq 2$, there exists a $C^{r}$-open and dense subset $\mathcal{R}_{\varphi, \Lambda} \subset C^{r}(M, \mathbb{R})$ such that

$$
\operatorname{dim}(L(\Lambda, f) \cap(-\infty, t))=\operatorname{dim}(M(\Lambda, f) \cap(-\infty, t))
$$

is a continuous function of $t$ whenever $f \in \mathcal{R}_{\varphi, \Lambda}$.

Still concerning the beginning of the dynamical spectra, the second author [Mor20] proved that, for typical pairs $(f, \Lambda)$ as above, the minima of the corresponding Lagrange

\footnotetext{
${ }^{14}$ This is a compact, $\varphi$-invariant, uniformly hyperbolic set of saddle type; cf. Hasselblatt-Katok's book [KH95].

${ }^{15}$ I.e., if $U_{0}$ is a neighborhood of $\Lambda_{0}$ such that $\Lambda_{0}=\bigcap_{n \in \mathbb{Z}} \varphi_{0}^{n}\left(U_{0}\right)$, then $\mathcal{U}$ is taken small enough so that $\Lambda=\bigcap_{n \in \mathbb{Z}} \varphi^{n}\left(U_{0}\right)$ still is a horseshoe for any $\varphi \in \mathcal{U}$.
}

and Markov dynamical spectra coincide and are given by the image of a periodic point of the dynamics by the real function, solving a question by Yoccoz.

Recently, Lima and the second author, ${ }^{16}$ proved that, for typical pairs $(f, \Lambda)$ as in the previous paragraphs,

$$
\begin{aligned}
& \sup \{t \in \mathbb{R}: \operatorname{dim}(M(f, \Lambda) \cap(-\infty, t))<1\} \\
& =\inf \{t \in \mathbb{R}: \operatorname{int}(L(f, \Lambda)) \cap(-\infty, t) \neq \emptyset\},
\end{aligned}
$$

and, inspired by this result, they conjectured that the classical Lagrange spectrum must have nonempty interior right after the transition point where the classical Markov spectrum acquires Hausdorff dimension one, i.e.,

$$
\operatorname{int}(L) \cap\left(t_{1}, t_{1}+\epsilon\right) \neq \emptyset
$$

for all $\epsilon>0$, where $t_{1}=\inf \{t: \operatorname{dim}(M \cap[\sqrt{5}, t])=1\}$.

Concerning the ending of dynamical spectra associated to horseshoes, Romaña and the second author proved that if $\Lambda$ is a (not necessarily conservative) horseshoe associated to a $C^{2}$-diffeomorphism $\varphi$ such that $\operatorname{dim}(\Lambda)>1$, then there is, arbitrarily close to $\varphi$, a diffeomorphism $\varphi_{0}$ and a $C^{2}$-neighborhood $W$ of $\varphi_{0}$ such that, if $\Lambda_{\psi}$ denotes the continuation of $\Lambda$ associated to $\psi \in W$, there is an open and dense set $H_{\psi} \subset C^{1}(M, \mathbb{R})$ such that for all $f \in H_{\psi}$, we have

$$
\text { int } L\left(f, \Lambda_{\psi}\right) \neq \emptyset \text { and int } M\left(f, \Lambda_{\psi}\right) \neq \emptyset \text {. }
$$

Another direct generalization of the classical spectra for geodesic flows on negatively curved manifolds and moduli spaces of translation surfaces (along the lines of \$2.4) was studied by Maucourant, Paulin, Parkkonen, Artigiani, Delecroix, Hubert, Lelièvre, Marchese, and Ulcigrai (among others): in their respective settings, these authors showed that their spectra shared some properties of the classical spectra such as isolated minima and a Hall's ray. We refer the reader to our recent book with Lima and Romaña [LMMR20] (and the references therein) for more details about dynamical generalizations of the classical spectra.

\section{References}

[Arn94] Pierre Arnoux, Le codage du flot géodésique sur la surface modulaire, Enseign. Math. (2) 40 (1994), no. 1-2, 2948. MR1279059

[Bar94] Arthur Baragar, Integral solutions of Markoff-Hurwitz equations, J. Number Theory 49 (1994), no. 1, 27-44. MR1295950

[BGS16] Jean Bourgain, Alexander Gamburd, and Peter Sarnak, Markoff triples and strong approximation, C. R. Math. Acad. Sci. Paris 354 (2016), no. 2, 131-135. MR3456887

[Bom07] Enrico Bombieri, Continued fractions and the Markoff tree, Expo. Math. 25 (2007), no. 3, 187-213. MR2345177

\footnotetext{
${ }^{16}$ See the article "Phase transitions on the Markov and Lagrange dynamical spectra" to appear in Ann. Inst. H. Poincaré Anal. Non Linéaire. https: // doi.org/10.1016/j.anihpc.2020.11.007
} 
[Bow79] Rufus Bowen, Hausdorff dimension of quasicircles, Inst. Hautes Études Sci. Publ. Math. 50 (1979), 11-25. MR556580

[CF89] Thomas W. Cusick and Mary E. Flahive, The Markoff and Lagrange spectra, Mathematical Surveys and Monographs, vol. 30, American Mathematical Society, Providence, RI, 1989. MR1010419

[dAMY01] Carlos Gustavo T. de A. Moreira and JeanChristophe Yoccoz, Corrigendum: "Stable intersections of regular Cantor sets with large Hausdorff dimensions", Ann. of Math. (2) 154 (2001), no. 2, 527. MR1865980

[DMM20] Vincent Delecroix, Carlos Matheus, and Carlos Gustavo Moreira, Approximations of the Lagrange and Markov spectra, Math. Comp. 89 (2020), no. 325, 25212536. MR4109576

[GMR19] Alexander Gamburd, Michael Magee, and Ryan Ronan, An asymptotic formula for integer points on MarkoffHurwitz varieties, Ann. of Math. (2) 190 (2019), no. 3, 751809. MR4024562

[Gol03] William M. Goldman, The modular group action on real SL(2)-characters of a one-holed torus, Geom. Topol. 7 (2003), 443-486. MR2026539

[KH95] Anatole Katok and Boris Hasselblatt, Introduction to the modern theory of dynamical systems, Encyclopedia of Mathematics and its Applications, vol. 54, Cambridge University Press, Cambridge, 1995. With a supplementary chapter by Katok and Leonardo Mendoza. MR1326374

[LMMR20] Davi Lima, Carlos Matheus, Carlos G. Moreira, and Sergio Romaña, Classical and dynamical Markov and Lagrange spectra, World Scientific, 2020.

[MM19a] Carlos Matheus and Carlos Gustavo Moreira, Markov spectrum near Freiman's isolated points in $M \backslash L, \mathrm{~J}$. Number Theory 194 (2019), 390-408. MR3860483

[MM19b] Carlos Matheus and Carlos Gustavo Moreira, $\mathrm{HD}(M \backslash L)>0.353$, Acta Arith. 188 (2019), no. 2, 183208. MR3925086

[MM20] Carlos Matheus and Carlos Gustavo Moreira, Fractal geometry of the complement of Lagrange spectrum in Markov spectrum, Comment. Math. Helv. 95 (2020), no. 3, 593633. MR4152626

[Mor18] Carlos Gustavo Moreira, Geometric properties of the Markov and Lagrange spectra, Ann. of Math. (2) 188 (2018), no. 1, 145-170. MR3815461

[Mor20] Carlos Gustavo T. de A. Moreira, On the minima of Markov and Lagrange dynamical spectra, Astérisque 415 (2020), 45-59. Quelques aspects de la théorie des systèmes dynamiques: un hommage à Jean-Christophe Yoccoz. I. MR4142446

[PT93] Jacob Palis and Floris Takens, Hyperbolicity and sensitive chaotic dynamics at homoclinic bifurcations: Fractal dimensions and infinitely many attractors, Cambridge Studies in Advanced Mathematics, vol. 35, Cambridge University Press, Cambridge, 1993. MR1237641

[Zag82] Don Zagier, On the number of Markoff numbers below a given bound, Math. Comp. 39 (1982), no. 160, 709-723. MR669663

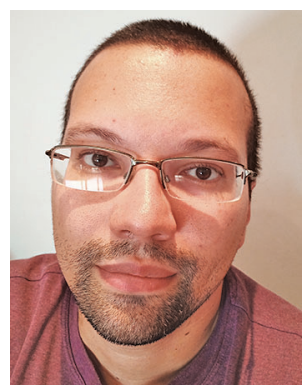

Carlos Matheus

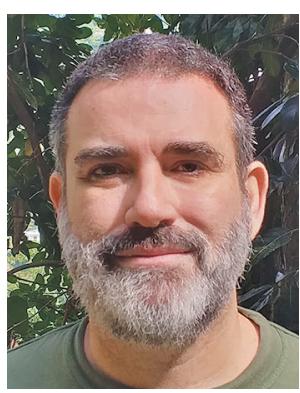

Carlos

Gustavo Moreira

\section{Credits}

Opening image is courtesy of Suebsiri via Getty.

Figure 2 is courtesy of Carlos Gustavo Moreira, Carlos Matheus, and Vincent Delecroix.

Photo of Carlos Matheus is courtesy of Carlos Matheus.

Photo of Carlos Gustavo Moreira is courtesy of Carlos Gustavo Moreira and Gabriela Brito Monti.

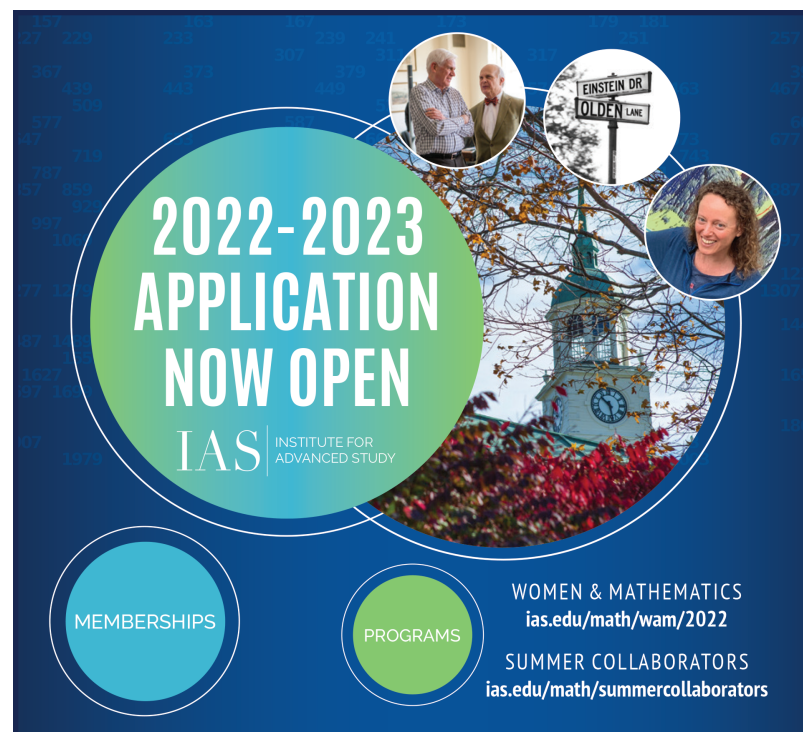

THE IAS SCHOOL OF MATHEMATICS welcomes applications from mathematicians $\&$ theoretical computer scientists at all career levels, and strongly encourages applications from women, minorities, and mid-career scientists ( $5-15$ years from Ph.D.). Competitive salaries, on-campus housing, and other resources are available for periods of 4-11 months for researchers in all mathematical subject areas. The School supports approximately 40 post-docs per year. In 2022-2023, there will be a special-year program, Dynamics, Additive Number Theory and Algebraic Geometry, led by Tamar Ziegler, Distinguished Visiting Professor; however, Membership will not be limited to mathematicians in this field.

To apply, submit your application at mathjobs.org by 12.1.21. For more information, please visit: ias.edu/math 